
INAP, 2017, 215 págs.

\title{
Jaime FERRI DURÁ \& Manuel BARROSO SEVILLANO (Coords.). Ciudadanía y servicios públicos locales: Un estudio en las comunidades autónomas de Madrid y Castilla y León. Madrid: INAP, 2017, 215 pp.
}

\author{
Mirian Keidel Fernández \\ Universidad Complutense de Madrid \\ mkeidel@ucm.es
}

\section{NOTA BIOGRÁFICA}

Graduada en Ciencias Políticas en 2017 y Máster en política Internacional: Estudios de Área y sector en 2018 por la Universidad Complutense de Madrid. Sus principales líneas de Investigación se centran en los cambios en actitudes políticas de la sociedad, la organización del poder político-económico y la transformación del Estado y la construcción democrática.

\section{RESUMEN}

El presente monográfico es el resultado de la coordinación por parte de Jaime Ferri Durá y Manuel Barroso de una investigación sobre la prestación de servicios públicos locales, en la que participan Juan C. Cuevas Lanchares, Eduardo Gutiérrez Díaz, Eliseo López Sánchez, Francisco J. Loscos, Luis Pérez Rodríguez y en la que también colaboran Tomás Villasante, Manuel Tamayo y Ernesto Carrillo tras haber colaborado en un Curso de verano de la UCM impartido en San Lorenzo de El Escorial en julio de 2016.

\section{PALABRAS CLAVE}

Prestación de servicios públicos.

\begin{abstract}
The present monographic is the result of the coordination of Jaime Ferri Durá and Manuel Barroso regarding the investigation concerning provisions of local public services. In addition, Juan C. Cuevas Lanchares, Eduardo Guitiérez Díaz, Eliseo López Sánchez, Francisco J. Loscos, Luis Pérez Rodríguez as well as Tomás Villasante, Manuel Tamayo and Ernesto Carrillo after collaborating in the summer course of the UCM in San Lorenzo July 2016.
\end{abstract}

\section{KEYWORDS}

Public service delivery. 
Esta investigación nace de la preocupación por la entonces recién aprobada Ley 27/2013, de 27 de diciembre 2013, de Racionalización y Sostenibilidad de la Administración Local, por lo que se estudian los servicios públicos que las entidades locales de las Comunidades Autónomas de Madrid y Castilla y León prestan en sus territorios y a sus poblaciones. En particular se analizan los servicios específicos que se prestan, cómo se prestan y el coste que tienen de acuerdo con muestras representativas estructuradas por número de habitantes en ambas comunidades. Dicha investigación es de especial interés porque a través de un minucioso análisis del actual modelo territorial de las Comunidades Autónomas de Castilla y León y Madrid y de la mencionada Ley 27/2013 de Racionalización y Sostenibilidad de la Administración Local, se vienen a desmontar algunos de los argumentos que defendía dicha norma. Así, el libro constituye una fuerte crítica a la pretendida reforma territorial llevada a cabo por aquel Gobierno.

Por otra parte, la investigación pretende aportar al área de la Ciencia Política y de la Administración un mejor conocimiento empírico sobre los actuales gobiernos y administraciones locales españolas, avanzando en el conocimiento de los servicios que debería prestar cada Ayuntamiento según sus capacidades.

Desde el punto de vista metodológico, cabe destacar la enorme labor de recopilar tanto datos cuantitativos como cualitativos. Para abordar de la forma más completa la información se estableció un cuestionario con tres grandes bloques de información ${ }^{1}$. La aplicación de este cuestionario a los sujetos de estudio se desarrollo de forma on-line, enviando el cuestionario tanto a responsables políticos (alcaldes o presidentes de diputación), como a funcionariales de todos los municipios que componen el universo, con el fin de analizar la dispersión en la distribución, así como la capacidad explicativa de las variables independientes, según el tamaño de la población y el tipo de puesto que ocupa el encuestado. Para el envío del cuestionario se realizó un muestreo de los municipios tanto de la Comunidad de Madrid como de la de Castilla y León.

Por otra parte, se organizó un grupo de discusión, con representantes de diferentes ayuntamientos de la Comunidad de Madrid para obtener de forma más cualitativa datos de interés, permitiendo completar la fuente cuantitativa. El grupo de discusión estuvo monitorizado por los profesores Jaime Ferri, y Luis Pérez.

Los datos de las fuentes cuantitativas de la encuesta fueron explotados mediante un análisis de estadística descriptica y los datos cualitativos del grupo de discusión fueron explotando con una análisis de discurso y contenido y el contraste de las narraciones de los distintos participantes ante los temas que iban introduciendo los monitores del grupo. Por último, en la tercera parte del libro se aportaron también conocimientos obtenidos en el Curso de verano de la UCM².

Como se puede observar, los datos que se aportan en este informe son suficientemente explicativos y tiene validez para los municipios contemplados en la investigación.

En cuanto a la estructura del libro, el libro esta compuesto por tres partes, en la primera parte del libro se realiza una aproximación al objeto de estudio, se plantean los objetivos las hipótesis del trabajo y la metodología que se ha seguido en la investigación.

En esta primera parte se analiza el antiguo modelo de reparto de competencias y la posterior la Ley $27 / 2013$ desde el marco jurídico, estableciendo lo que significa dicha ley y las implicaciones que tiene su aplicación.

El argumento por el cual se aprobó la Ley $27 / 2013$ se basa en la complejidad del antiguo modelo de administración territorial en lo que se refería a las entidades locales y que ello generaba, al menos, dos consecuencias indeseables:

Por un lado, este modelo dispersaba la responsabilidad de los gobiernos locales en el ejercicio de sus funciones y consolidaba una fusión respecto al ámbito competencial de la administración lo que tenía como consecuencia un cierto desconocimiento del ciudadano en torno a la administración responsable de cada servicio público. Por otra parte, generaba repercusiones negativas en las haciendas locales. Por lo que la citada ley tiene como objetivo asegurar que la administración local no vuelva a despeñar funciones correspondientes a competencias que estén atribuidas por la ley y para las que no cuenten con un adecuado respaldo

1 (a) Qué servicios prestan las entidades locales en el ámbito de competencias establecidas por la Ley 27/2013.

(b) Cómo se prestan, con la pretensión de identificar tato los mecanismos de coordinación, cooperación o asociación como la forma de gestión de los servicios locales.

(c) Cuál es su coste o volumen de recursos destinados a la financiación del servicio el presupuesto correspondiente al año 2013.

2 Ciudadanía y servicios públicos en los nuevos ayuntamientos, curso de verano de la Universidad Complutense que se desarroIlo del 11 al 15 de julio de 2016 en San Lorenzo de El Escorial. Esta iniciativa dio lugar a la continuidad de la temática de la investigación ya realizada, quiso acercar las diferentes visiones de académicos/as, representantes políticos, técnicos de la administración de la ciudadanía en general. 
GAPP. Nueva Época - N. ${ }^{\circ}$ 20, noviembre 2018 - ISSN: 1989-8991 - DOI: 10.24965/gapp.v0i20.10542 - [Págs. 164-167] Número monográfico - Gobernanza y políticas de desarrollo urbano: teoría y práctica

Jaime FERRI DURÁ y Manuel BARROSO SEVILLANO (coords.). Ciudadanía y servicios públicos locales: Un estudio en..

Mirian Keidel Fernández

presupuestario. Sin embargo, desde una perspectiva crítica, como indican los autores Juan C. Cuevas et. al. (2017) uno de los objetivos clave que persigue la reforma de la administración local consiste en el fomento de la iniciativa económica privada.

Por otra parte, no parece que la deuda pública local tenga suficiente peso para justificar la reforma de este nivel territorial de Administración, sino que este indicador apuntaría hacia la revisión de la organización institucional y del marco competencial de las Comunidades Autónomas y fundamentalmente, de la Administración General del Estado.

Una vez analizadas las implicaciones que tiene la nueva Ley 27/2013 en la segunda parte del libro, se estudian los ingresos de las Administraciones Locales y sus necesidades de gasto en contexto con la crisis económico financiera en España. En lo referente a las modalidades de gestión de los servicios públicos prestados en las entidades locales de ambas comunidades autónomas se concluye que existen pocas diferencias entre los ayuntamientos de Castilla y León y de la Comunidad de Madrid. Las diferencias de ciertos servicios, como agua y transporte urbano se deben a características sociodemográficas, pero son puntuales y poco significativas.

Otra diferencia destacable en la investigación, es que en la comunidad de Madrid los ayuntamientos suelen asumir más prestaciones de servicios, en comparación con los ayuntamientos de Castilla y León, donde los niveles de no prestación de ciertos servicios es elevada. Según los investigadores esto se debe al tamaño del municipio.

Siendo ésta la principal consecuencia de esta variable independiente y el principal hallazgo de la investigación: «el tamaño condiciona que se asuma un servicio o no, pero no condiciona que se gestione de una forma u otra.»

Por otro lado, en lo referente a la reforma del sistema financiero municipal, los autores destacan que la evolución del gasto público municipal en el periodo 2005-2013 muestra un comportamiento cíclico caracterizado por una elevada elasticidad, que se manifiesta especialmente en los gastos de personal y en los de inversión municipal. Desde el punto de vista de la clasificación funcional, las políticas de gasto con mayor elasticidad son las de vivienda y urbanismo, educación, servicios sociales y sanidad que confirman su naturaleza de «bienes superiores».

En lo referido al sistema de financiación de los municipios españoles los investigadores afirman que este descansa fundamentalmente sobre la recaudación de sus tributos propios y las transferencias recibidas del Estado, de manera incondicionada, en concepto de participación en los ingresos tributarios del Estado y, para los municipios de mayor tamaño, la cesión de un porcentaje de la recaudación de los principales tributos. La evolución del sistema de financiación municipal durante el periodo de análisis ha puesto de manifiesto una gran heterogeneidad en su grado de respuesta a la crisis económica, por lo que los investigadores apunta a una necesaria revisión para mejorar su eficiencia en el cumplimiento de los principios constitucionales de suficiencia y autonomía, y para desarrollar mecanismos efectivos de nivelación que articulen su contribución al logro de los objetivos de equidad y solidaridad.

Desde un punto de vista fundamentalmente normativo, tomando como pretexto el control del gasto público local con el fin de atenuar una vinculación entre ambos niveles de Administración se considera que se produce una reordenación territorial del poder, que se desarrolla una reafirmación del poder del Estado frente a las comunidades autónomas.

Por último en esta segunda parte del libro, en cuanto a las relaciones intergubernamentales, en la Comunidad de Madrid se pueden observar conflictos de competencia, pues en el caso de que las entidades no ejerzan las competencias o no presten los servicios a los que por ley estén obligadas, cuando su financiación esté legalmente garantizada y dicho incumplimiento afecte al ejercicio de las competencias de la Comunidad de Madrid, esta podrá subrogarse en su ejecución. En el caso de las entidades locales de la Comunidad de Castilla y León, se puede comprobar que, en la medida que aumenta el nivel de población, para la prestación de servicios públicos municipales, la relación entre tamaño poblacional y prestación de servicios se pierde, y los servicios los prestan los ayuntamientos aunque no se corresponda su población con la estipulada, para prestarlos, con fijada ley.

En lo que respecta a los servicios públicos prestados en los municipios en función de la delegación de la ejecución de las competencias del Estado y de la Comunidad Autónoma de Castilla y León, no existe una sistemática en la delegación de la ejecución de esas competencias o no existe esa delegación y se prestan esos servicios en función de lo que las juntas de gobierno Municipal de cada municipio hayan ido desarrollando a lo largo del tiempo y por tanto, no teniendo nada que ver con las poblaciones comprendidas entre 5.000 y 20.000 habitantes. 
GAPP. Nueva Época - N. 20, noviembre 2018 - ISSN: 1989-8991 - DOI: 10.24965/gapp.v0i20.10542 - [Págs. 164-167] Número monográfico - Gobernanza y políticas de desarrollo urbano: teoría y práctica

Jaime FERRI DURÁ y Manuel BARROSO SEVILLANO (coords.). Ciudadanía y servicios públicos locales: Un estudio en..

Por último me gustaría destacar la importancia de la tercera parte del libro, en la cual se plantea formulas alternativas de planificación del territorio y los servicios públicos de forma más integral y participativa, analizando las diferentes experiencias de otros países. Desde distintos enfoques se contribuye a un debate sobre una gestión integral de los servicios públicos, con democracias de iniciativas, que incluyen los presupuestos participativos, pero que van más allá con otras formas que les dan un mayor alcance. Se presentan algunas escaleras y esquemas de varios circuitos para la toma de decisiones, planificación y seguimiento de los procesos, lo que permite presentar un sistema integral para la gestión de los municipios del cambio.

En este último apartado también se apunta a que tanto los cambios señalados, como sus impactos y los instrumentos político-institucionales abordados son de carácter estratégico, afectan al menos a los Gobiernos de las ciudades de los sistemas democráticos y forman parte de tendencias de larga duración.

Ante estas conclusiones los autores terminan destacando la importancia de una propuesta de gestión pública que tome como punto de partida los valores públicos, siendo el ideal del interés público su objetivo final. En últimas instancias buscan una gobernanza urbana éxitos en la promoción de los valores públicos y en el interés de la ciudad o, al menos, en la que el mercado y el Estado cometan menos fallos posibles.

A pesar de la dificultad de la investigación, dada la peculiaridad de las dos Comunidades autónomas analizadas y la complejidad de la planificación territorial administrativa, el análisis y las posteriores conclusiones se presentan de forma muy clara, facilitando la comprensión al lector. 